\title{
NATURA E ALTERITÀ ANCORA SULL'ARIA DEL TASSO DI GIUSEPPE TARTINI
}

\author{
IVANO CAVALLINI
}

Università di Palermo

Izvleček: Giuseppe Tartini je štiri stavke svojih Abstract: Giuseppe Tartini based four moveviolinskih sonat osnoval na ljudski melodiji ments of his violin sonatas on the popular theme Aria del Tasso. Izraz popolare, ki ga uporablja Aria del Tasso. The term popolare in Tartini's $v$ svojih spisih, je sinonim za preprosto in pre- writings is equivalent to simple, and simplicity prostost je glavna značilnost narave. Tartini is the main feature of nature. Tartini defines opredeljuje harmonijo kot naravni fenomen, harmony as a phenomenon of nature imbued with prežet s splošnimi vrednotami, ki so osnova vsake a universal value, on which both traditional and ljudske ali umetne glasbe. Sprejema raznolikost art music are founded. He accepts the diversity of kulturnih oblik, ki sobivajo v okviru vzporedne cultural forms coexisting within the framework of druge narave, in s tem priznava drugačnost a parallel second nature, thus recognizing alterkot pojavno kategorijo. Iz te dvojnosti pa sledi ity as an emerging category. This dualism implies trk ratia in sensusa, ki njuno dvojnost skoraj a clash between ratio and sensus, turning their spreminja $v$ naravo in čustvovanje. opposition almost into one of nature and nurture.

Ključne besede: Giuseppe Tartini, narava, har- Keywords: Giuseppe Tartini, nature, harmony, monija, ljudske pesmi, drugačnost. folk songs, alterity.

Esser giovani vuol dire esser primitivi, esser rimasti vicini alle scaturigini della vita, $[\ldots]$ osare quello che gli altri non hanno l'ardire di fare, cioè di rituffarsi nella elementarità [Deutschlin a Adrian Leverkühn].

Thomas Mann, Doctor Faustus. La vita del compositore tedesco Adrian Leverkühn narrata da un amico (trad. di Ervino Pocar, Milano: Mondadori, 1996, 135)

Negli scritti di Giuseppe Tartini il termine natura è sinonimo di verità. Equivale alla semplicità incontaminata cui si contrappone l'artificio umano, definibile come un apporto culturale distinto dalla verità di natura. In questa categoria Tartini colloca il canto popolare, la musica degli antichi e i suoni dei primitivi. Tuttavia, per disambiguare il lemma da una polisemia fuorviante, è d'uopo ricordare che nelle testimonianze dei suoi estimatori la natura coinvolge l'espressione di sentimenti autentici traslati in note (così Francesco 
Algarotti, Jean d'Alembert, Gianrinaldo Carli). ${ }^{1}$ Il violinista, estraneo alle insidiose querele su vero e verisimile, si riferisce invece alle manifestazioni canore che fioriscono in modo spontaneo, ivi compresa l'Aria del Tasso dei gondolieri veneziani, della quale penso sia inutile chiedersi se le sue origini siano propriamente folkloriche. Ritenuta all'epoca un esempio di canto naturale, essa riceve la classificazione di melodia del popolo in quanto elementare nella struttura e patrimonio di uno strato sociale non progredito e quindi più vicino alla civiltà dei primordi.

La particolare attenzione di Tartini verso il popolo non può essere liquidata come un episodio prossimo all'esotismo e nemmeno sopravvalutata come un caso in anticipo sulle future applicazioni dell'antropologia alla musica. Essa si rapporta a vari modelli di alterità, come si è detto oltre al popolo il primitivo e l'antico, e si collega in via prioritaria al concetto di purezza primigenia, esaltata quale indizio di naturalità. A questo riguardo va detto che Tartini non si è mai occupato in modo sistematico del problema. Al contrario, il dubbio che si insinua nella sua mente circa un presunto accordo tra ragione e mondo reale fa generare una serie di pensieri frammentari sull'altro, da valutare con circospezione poiché non accedono mai alla dimensione speculativa del trattato di estetica, né alla trascrizione provvista di commento etnografico.

Nel passato Pierluigi Petrobelli, Roberto Leydi e Pierpaolo Polzonetti hanno affrontato il tema dell'oralità per individuare le fonti a cui ha attinto il maestro, e verificare se egli le ha riprodotte fedelmente, o se ha ricreato i processi compositivi caratteristici del folklore. ${ }^{2}$ Premesso che le due possibilità non si escludono a vicenda, lo scopo del mio intervento esula da quel tipo di esame. A parte l'Aria del Tasso, infatti, le prime registrazioni su pentagramma dei canti folklorici dell'Istria, italiani, sloveni e croati, risalgono al ventesimo secolo. Anche ammettendo il carattere conservativo di quel repertorio, l'assenza di pezzi in notazione contemporanei a Tartini rende meno efficace il confronto. A questo punto il fuoco della ricerca si può spostare sulle polarità stabilite nella prosa del maestro dai termini natura, ragione, armonia vs. uso, costume, sentimento, assuefazione, istituzione, nazione. Tutti assieme questi vocaboli concorrono a disegnare un quadro di riferimento, entro il quale si aprono un varco tre alterità, a iniziare da quella folklorica ancora allo stato aurorale. Per dirla in altro modo, qualsiasi disamina a partire dal popolo è destinata a fallire l'obiettivo, se non si tratta questo termine come un soggetto che vive in simbiosi con quel lessico, dal quale trae il proprio nutrimento logico. Tale subordinazione implica il ricorso ad alcuni sinonimi, indispensabili per avviare una corretta decifrazione del significato, cui si può affiancare un ulteriore paragone con le proposizioni dei letterati coevi a Tartini, per una migliore comprensione del mutamento di prospettiva teorica che

1 In questo articolo il termine nazione è sempre virgolettato per evitare di doverlo confondere con il termine popolo. Quest'ultimo, nella lingua italiana corrente, ha la duplice valenza di nazione e di classe; per esemplificare: il popolo italiano, ossia tutti gli italiani; nel secondo caso: il popolo distinto dalla nobiltà e dalla borghesia. Nella prosa di Tartini il termine popolo si avvicina di più all'odierno significato di cultura subalterna all'interno della nazione; al contempo il canto popolare, a causa dei suoi aspetti conservativi, veri o presunti, acquista il valore di generico canto di tradizione. Su Tartini e i suoi estimatori cfr. Cavallini, "Genio, imitazione."

2 Petrobelli, "Tartini and Folk Music;" Id., Tartini, le sue idee, 101-108; Leydi, "Erminia monta in gondola;" Polzonetti, Tartini e la musica secondo natura. 
scaturisce dalle sue meditazioni informali. Da una rassicurante strategia, ove si accordano meccanicisticamente ragione e senso in nome dell'armonia (ratio = sensus), e nell'ambito del senso si insinua una timida presa di coscienza della musica non colta, egli indulge nella tormentata dialettica tra natura e culture altre, ossia tra una pretesa legge universale dell'armonia e gli usi particolari che mettono in forse la sua capacità normativa (natura/ mathesis vs. culture). Poi, con una diversione inattesa, Tartini predispone l'incontro tra natura e cultura sul piano del genere diatonico, anche se ciò lo costringe a evitare i presupposti di una ratio autonoma, svincolata dagli arbítri dell'operare umano. In conseguenza di tale svolta la natura si identifica nell'usanza e trascolora nel vasto campionario delle musiche create dall'antichità sino ai suoi giorni (natura i.e. cultura).

Fermi allo stadio primigenio, al cospetto della musica colta, sono per Tartini il canto di popolo, indistintamente urbano o contadino, la cosiddetta musica dei consessi umani non evoluti e la musica degli antichi greci, giudicata schietta al cospetto della moderna artificiosa. Oggi, come nel Settecento, ciò che apparenta la musica del popolo con quella degli antichi è la trasmissione orale, cui Tartini non concede spazio nella sua teoresi, e non certo il paradigma della semplicità. Ovviamente, in mancanza di una preparazione antropologica dell'intero mondo lettré, era facile cadere in simili fraintendimenti, soprattutto per la larga diffusione nei diversi strati sociali di scheletri di melodia, o di giri armonici essenziali, su cui si sbarcavano da tempo immemore le stanze di sonetti, canzoni e strambotti. Solo la generazione successiva si avventerà sull'oralità in Omero, dopo lo scalpore suscitato dai canti di Ossian in un'Inghilterra occupata a darsi un'identità nazionale attraverso i poemi gaelici del terzo secolo, sulle cui tracce si porrà la Bardendichtung nei paesi di lingua tedesca. L'annullamento della distanza temporale tra questi fenomeni permetterà di contemplare una parentela diversamente impossibile a istituirsi. Anche se con modalità tanto contorte e difficili da spiegare, tale svolta non sarebbe avvenuta senza il contributo di un manipolo di precursori, Tartini compreso, i quali iniziarono a introdurre i semi del dualismo folk/musica d'arte nelle categorie naturale/artificiale. ${ }^{3}$

Per contestualizzare le pulsioni antropologiche e antiquarie del piranese è doveroso seguire l'ordine cronologico degli asserti da porre al vaglio critico. Diversamente dai viaggiatori, all'epoca gli unici a documentare i fenomeni qualificabili come musica di tradizione, Tartini chiama in causa le musiche altre allo scopo di fissare i precetti validi per qualsiasi civiltà. Nel Trattato di musica secondo la vera scienza dell'armonia (1754) prende in considerazione "i balli contadineschi diretti da un cembalo, strumento [...] senza suono" (i.e. un tamburello), per sostenere che alcuni moduli ritmici attraversano sia il confine tra colto e popolare, sia tra le "nazioni". "Essendo io convinto da un fatto di natura", scrive l'autore, "e persuaso che in eguali circostanze lo stesso debba succedere appresso qualunque nazione, devo credere alla mia ragione appoggiata e provata da un fatto che mi libera da qualunque sospetto di pregiudizio". ${ }^{4}$ Trovata la chiave speculativa come nelle scienze esatte, egli osserva che in luoghi diversi, ma in presenza di fattori ambientali analoghi, il risultato non cambia. L'approccio razionale applicato alla dimensione umana

3 Gelbart, The Invention of "Folk Music". L'autore si intrattiene su questa svolta culturale, con particolare attenzione ai primi saggi sulla musica scozzese nel diciottesimo secolo, poiché testimoniano l'insorgere dei temi folklorici a fronte di una radicale conversione del termine natura.

4 Tartini, Trattato di musica [ed. moderna], 199. 
previene a sua detta dal viziare il giudizio attraverso l'impressione personale, soggetta all'influenza di sensazioni volatili fuori da qualsiasi regola. Metodo felicemente rinverdito da Newton, il quale, dopo Galileo e Huygens, aveva proceduto alla "matematizzazione" della filosofia naturale, basandosi sull'universalità delle forze esistenti nel creato. La traduzione latina della Opticks (1706), destinata al pubblico europeo, contiene l'invito a trasporre quel metodo in altri campi della conoscenza, come si può intuire dal passo che predica il risalire dagli effetti alle cause e la ricostruzione dal particolare al generale: "Methodus analytica est experimenta capere, phaenomena observare; indeque ex rebus compositis rationatione colligere simplices: motibus, vires moventes; et in universum, ex effectis, causis; ex causis particolaribus, generales." 5

Nell'ottica di una vagheggiata unità dei saperi si situa pure la fiducia di Tartini nel procedere per riduzione a pochi principi. Onde arrivare alla definizione della norma antropologica egli parte dallo studio dell'armonia. Guardata nel suo sviluppo secolare, la disciplina attesta che tra i "sistemi" praticati in Occidente l'unico a essere rimasto immutato è il genere diatonico. Questo, infatti, è il solo a ricevere una decifrazione matematica grazie ai calcoli che predeterminano le leggi del suono sin dai tempi di Pitagora, cui si allegano altre conferme desunte dall'acustica: "il solo sistema diatonico si regge da sé ed è sufficiente a sé stesso; e però si è usato da’ greci, dagli antichi italiani e si usa da noi quando si vuole a tutto rigore della sua natura e costituzione". ${ }^{6}$

La soluzione offerta dal compositore enfatizza la costruzione delle scale seguendo un criterio scientifico assoluto a discapito della varietà. Eppure non gli era ignoto il fatto che i popoli possiedono musiche diverse in quanto sviluppate su libere convenzioni, da considerare, secondo il suo pensiero, quali atti arbitrari divenuti tradizione. Pur cosciente dei limiti che si impongono in materia d'arte declinata secondo l'estro delle "nazioni", Tartini si rivolge al quid unificante, ossia a un canone trasversale ai luoghi, ma soprattutto ai tempi, essendo egli uno studioso di teoria. In questo senso il violinista persegue l'ideale di estendere a qualsiasi prassi musicale un criterio di misurazione matematica.? Tuttavia, in un altro passaggio del Trattato non esita a mitigare gli effetti dell'impianto assiologico, accogliendo accanto ai tratti comuni quelli che dividono le culture. Oltre al fondamento scientifico su cui coordinare ogni valutazione, agiscono per Tartini altre costanti come la lingua e l'indole dei popoli, le quali contribuiscono a diversificare le civiltà musicali senza negare la medesima matrice armonica. A questo punto il maestro mira a confermare l'esistenza di una grammatica diffusa che comprende, in via astratta, tutte le espressioni sonore. Non rinuncia però a sottolineare che "quando si proponga lo stesso fine de' greci, siamo affatto lontani dalla possibilità di ottenerlo co' nostri mezzi. [...] Vi si oppone la nostra cantilena, come modulata secondo l'arte nostra, come sciolta da qualunque ragguaglio materiale e formale alla prosodia." Occorre "conformità di idee rispetto al costume", ammette infine il piranese, affinché l'elemento divenuto esogeno a causa dello iato spazio-temporale si integri nella cultura corrente.

\footnotetext{
5 Optice: sive de reflexionibus, 347; Hall, "La matematica."

6 Tartini, Trattato di musica, 221.

$7 \mathrm{Su}$ questo aspetto cfr. l'introduzione di Gilson alla versione italiana di Descartes, Discorso sul metodo, 1-19.

8 Tartini, Trattato di musica, 246.
} 
Alla continuità tra legge e utenza, le regole e il loro uso, ora sembra frapporsi un limes difficile da oltrepassare. Lo accerta il paradosso cronologico degli antichi greci intenti a intonare le cantilene dei dalmati. Mediante lo scambio fittizio Tartini spiega il concetto di tradizione, tradotto con i sinonimi "assuefazione" e "costume", giacché, rispetto a una genesi indifferenziata secondo la teoria creazionista della chiesa, è intervenuta la ripartizione delle civiltà. Nel rivolgersi direttamente al suo mecenate e amico, il conte Decio Agostino Trento, scrive:

Le mie proposizioni sono dedotte dalle osservazioni di natura, comune a' greci e a noi. La loro precisione [= coincidenza, $\mathrm{NdA}$ ], dunque, non nasce intrinsecamente dalla loro deduzione, ma dalla adattazione a' modi antichi e moderni tra loro comparata [...]. Si richiede in chi ascolta l'animo disposto in specie secondo diversi rispetti, e sono: assuefazioni a quel tal genere di musica. Son certo che in tal rispetto il migliore tra greci antichi potrebbe, senza ottenere il suo fine, cantare a talento alla odierna dalmata nazione, la di cui musica non ha intervalli determinati, ma è un continuo di voce, protratto a discrezione in grave e acuto. Conformità di idee rispetto al costume, o rimarrà indifferente, o se sono contrarie, più che mosso sarà ributtato. Son certo che rappresentato ad un cannibale un oggetto, nel costume il più tragico, non aborrirà, ma goderà dell'oggetto. ${ }^{9}$

Poco innanzi, sempre a proposito di tale dicotomia, arguisce che "vi sono popoli e nazioni intiere di moto per natura tardo, altri di veloce, altri di temperato". ${ }^{10} \mathrm{E}$ pare di leggere il settimo capitolo del Trattato dell'imitazione di Antonio Conti, il quale, da empirista conoscitore della fisiatria, annota che "la divisione delle voci, l'agilità, gl'increspamenti dipendono in gran parte dalla qualità del clima, che rende più o meno mobili gli anelli della trachea". ${ }^{11}$ L'abate veneto, amico di Newton e membro della Royal Society, ritiene non esservi contraddizione tra l'armonia e la musica variamente connotata dei consessi umani. ${ }^{12}$ L'armonia non è una convenzione creata ad arte, bensì un requisito della natura indipendente dall'azione dei compositori e si esplica nel gradevole ascolto delle consonanze semplici. In difesa della congruenza di sensus e ratio egli mette in campo l'autorità di Descartes, il quale, nel Compendium musicae (1618), "non ha dedotto queste regole da principi arbitrari, ma dalle proprietà del diletto che ama il senso e su queste stesse proprietà sono dedotte l'altre regole accennate dal Mersenno". ${ }^{13}$ Ciò non toglie che le cause ambientali concorrano a rendere singolari le musiche delle "nazioni", per cui anche quando esse seguono "le proporzioni numeriche dei toni, ognuna adatta quei toni

9 Ibid., 247.

${ }^{10}$ Ibid.

${ }^{11}$ Conti, Prose e poesie, 115-116; raccolta postuma in forma di riassunti degli scritti dell'autore curata da Giuseppe Toaldo (il primo volume è del 1739).

${ }^{12}$ Conti rientrò a Padova nel 1726 e vi morì nel 1749. Le sue idee sulla coniugazione tra piacere estetico e processi gnoseologici, nonché la vasta rete di rapporti scientifici ed artistici con i personaggi più in vista di Francia, Italia e Inghilterra, potevano essere di stimolo a Tartini, anche se non è documentata alcuna frequentazione tra i due pur residenti nella stessa città. Cfr. Badaloni, Antonio Conti e l'art. di Gronda, "Conti, Antonio (Schinella)."

${ }^{13}$ Conti, Prose e poesie, 119. Si riferisce al musicografo Marin Mersenne. 
al suono che l'è proprio". ${ }^{14}$ Esempio ne sia la musica degli orientali, i quali, "avvezzi ai suoni delle lor lingue gutturali, nulla trovano d'imitativo ne i suoni delle nostre". ${ }^{15}$

A parte la coincidenza con le annotazioni di Conti, circa il supposto equilibrio tra gli universali e "i costumi singolari", viene da chiedersi per quale ragione Tartini evochi nel suo Trattato la "dalmata nazione" al posto del popolo per alludere a manifestazioni canore tanto diverse. Con tutta evidenza egli pensava a una civiltà geograficamente vicina, e coabitante con l'italiana, ma assai discosta da quest'ultima. Non è dato infatti confondere la musica dei dalmati, che per Tartini "non ha intervalli determinati", con la musica dei suoi istroveneti. Con la locuzione "odierna dalmata nazione" egli pone un discrimine etnico, che indica una eredità canora differente da quella delle regioni finitime. Una diversità che induce a pensare al canto glagolitico migrato in alcune zone della natia Istria (a Capodistria, città contigua a Pirano, vi erano i monaci glagoljaši che servivano sloveni e croati), o alle pjesme profane, ossia i canti di tradizione orale appartenenti ai soli slavofoni, o alla istarska ljestvica a sei toni, scala istriana non temperata dei croati. ${ }^{16}$ A tale riguardo, per non incorrere in errori di tipo onomastico, sino al diciannovesimo secolo per designare gli slavi autoctoni della costa adriatica si adoperavano i sostantivati illirici o dalmati, giacché l'etnonimo croato era valido principalmente per gli abitanti dell'attuale Croazia del nord.

Nel corso degli anni Cinquanta Tartini provò cautamente a configurare un paradigma che servisse a rendere concordi l'esperienza uditiva con la matematica e i ritrovati della fisica. Sconfortato da altrettante prove contrarie, desunte dalla storia ed esibite nello spazio, nel 1760 egli ruppe ogni indugio riconoscendo l'autonomia culturale dei gruppi umani. La risposta a una missiva del conte Giordano Riccati, collezionata nel Commercio di lettere intorno ai principj dell'armonia, è emblematica di questo radicale cambiamento di opinione:

Per altro se il signor Conte riflette seriamente ad un solo fatto, ed è la nostra musica tanto diversa da quella de' dalmati, turchi, chinesi, americani etc., è forza che da sé si confonda, perché in qual maniera potrà il signor Conte convincer gli altri della imperfezione della loro, della perfezione della nostra musica? non certamente in forza di orecchio e di esperienza, perché in forza di orecchio e di esperienza han fondato la loro, la quale a confronto appunto di esperienza credono migliore della nostra, come noi crediamo la nostra della loro migliore. Dunque o sono tutte a egual condizione, o è forza che vi sia un diverso principio, e di altra categoria che provi e dimostri la imperfezione delle altre, la perfezione della nostra. Prova sì fatta non può venire dal sentimento, perché anzi o è viziato in noi, o negli altri dal'uso fatto natura, e però qual si trova bisogna distruggerlo. E che mai potrà distrugger un sentimento formato in natura, se non che una ragione dimostrativa della falsità e vizio del sentimento? ${ }^{17}$

Il fatto che egli citi dalmati, turchi, cinesi e americani non solleva nuove questioni, come si è creduto, in merito al folklore piuttosto che alla musica colta o rituale di quelle

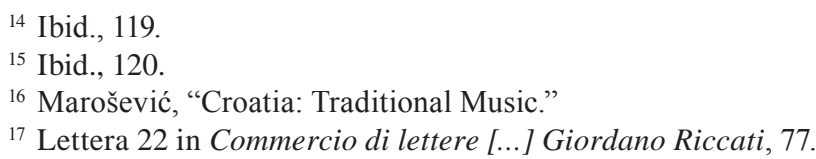


genti. Il termine "nazione" non può essere confuso in questo passo della lettera con il termine popolo, inteso quale classe avvezza alla musica vernacolare. Le informazioni fornite su quelle etnie dalla letteratura odeporica e dalla storiografia erano troppo sommarie per consentire a Tartini di distinguere nel turco o nell'americano il colto dal demotico, e ciò anche ammettendo l'esistenza di simili categorie tra gli ottomani o gli indiani d'America. Non a caso egli parla di "nostra musica", per alludere ai generi alti senza porsi il problema dell'imparagonabilità con i popolari. Inoltre, anche questo passaggio pare calcato su un analogo di Conti, il quale, al contrario, non scorge alcuna frattura tra l'armonia universale e le musiche delle "nazioni":

La musica italiana [...] che fa tanto effetto sull'animo degl'italiani, non ne fa alcuno sull'animo de' francesi [...] e lo stesso può dirsi della musica turca, chinese, americana e d'altre, perché ogni popolo separato da' monti e da' mari, come ha una lingua, così ha una musica propria. [...] Ho più volte osservato ne' miei viaggi che la qualità del suono della voce degli uomini e delle donne è proporzionata alla loro sussistenza e al temperamento del corpo loro, e che perciò ogni nazione ha un suono determinato, col quale abbassa ed innalza le voci fino a un certo grado. Or la musica non fa che ridur a misura i suoni naturali, onde ogni nazione cantando, se ben conviene nel seguire le proporzioni numeriche de' tuoni musicali, però ognuna la veste del suono che l'è proprio. ${ }^{18}$

Tartini, grazie a un'intuizione rimarchevole e senza il sostegno di teoremi estetici, ribalta clamorosamente la dinamica che saldava quanto era ritenuto naturale per la scienza dell'armonia con quanto era conseguente allo sviluppo differenziato dei popoli. Egli rigetta qualsiasi paragone tra la musica eurocolta e le musiche altre, quando esso si basa sul semplice ascolto. Ogni "nazione" vanta una propria esperienza che si esplica nella diversità dello stile e degli assetti teorici, e potrebbe sentirsi superiore alla nostra se il giudizio si forma con il solo udito senza ponderare i fenomeni che comportano la diversità. Ciò non osta al fatto che si debba rinunciare alla fissazione di una ratio scientificamente comprovabile, alla quale può fare capo qualsivoglia prassi particolare. A quanto è dato intendere, il teorico non vede contraddizione alcuna tra la natura quale costume dei popoli e la natura fisica del suono, ma è costretto a mettere tra parentesi la priorità di un collegamento vincolante secondo la logica causa-effetto, e accettare la temporanea convivenza di due nature in attesa di trovare l'elemento esplicativo di tale diarchia. Questa fase critica, della cui durata non è agevole dire, mette in evidenza l'inabilità di Tartini a rassegnarsi all'idea che ambo le verità di natura non siano riconducibili all'unità. Per risolvere il problema della reciproca esclusione egli punterà in seguito a cambiare il procedimento scientifico: partendo dall'indagine storica ed empirica, cercherà di cogliere in un altro quid la forza coesiva per supplire alle carenze di un ragionamento che costringe entro il recinto del numero armonico anche quello che vi si oppone.

Giordano Riccati, il corrispondente epistolare degli anni Sessanta, si interroga a sua volta sull'effettivo legame esistente tra proporzioni e musica, e sostituisce ai principi matematici la percezione sensibile. L'orecchio, strumento perfetto donato all'uomo da Dio, sarebbe in grado di giudicare secondo valori oggettivi che non sono in contrasto con il numero pressoché infinito delle combinazioni sonore. Con una soluzione drastica,

${ }^{18}$ Conti, Prose e poesie, 119. 
che tende a spostare il dibattito sul piano dell'estetica, il nobile trevigiano supera così l'ostacolo delle culture. Egli reputa essere "il bello musicale" non già frutto "d'opinione e d'abitudine", bensì un bello di natura, anche se passibile di innumerevoli adattamenti. ${ }^{19} \mathrm{Un}$ modo di risolvere l'annosa questione che non è congeniale a Tartini e non lenisce di certo il suo assillo. Per di più non è dissimile dal ragionamento di Denis Diderot, esplanato con cura nei Principes généraux d'acoustique del 1748. Secondo l'opinione del philosophe l'acustica, l'orecchio, la fantasia e la geografia umana non sono determinanti nel definire "le plaisir musical", che risiede nella "perception des rapports des sons". ${ }^{20}$ Quale che sia l'arte, il giudizio sul bello dipende da una simmetria che gli uomini colgono a livello sensoriale, ma che agisce come un canone rigoroso a dispetto della soggettività e delle mutazioni secolari: "le plaisir, en général, consiste dans la perception des rapports". Un precetto inaccettabile se applicato con rigore scientifico, giacché avrebbe portato ad ammettere la diseguaglianza tra i gruppi umani sulla base delle molteplici simmetrie. Diderot non se ne cura e nel 1752, con la voce Beau Joli dell'Encyclopédie, si compiace di rovesciare l'ordine dei fattori nel ripetere con la stessa convinzione che "tout ce qui peut exciter en nous la perception des rapports est beau". ${ }^{21}$

Le considerazioni di Riccati e Diderot ineriscono a un'estetica del gusto, ove "il bello essenziale", "il bello naturale" e "il bello artificiale" si traducono con la ratio a priori, il mondo materiale e l'opinabile dell'operare umano. In Tartini ragione e natura si fondono in una datità commisurata alla scienza (e non a Dio); e sebbene "l'institution humaine" di Diderot coincida con le condizioni dettate dall'arte e dagli assetti civili, il nostro rimarrà sempre al di qua di una ideale linea di demarcazione, oltrepassata la quale iniziano le disquisizioni filosofiche su arte, gusto e imitazione. La sua sola ambizione è di asseverare l'esistenza di un fattore aggregante, da rintracciarsi nella natura-scienza stante la fallacia dei sensi. Il che non gli impedisce di abbracciare un concetto di cultura parcellizzata sulle attitudini dei popoli, la cui musica è dichiarata diversa a causa di un "uso fatto natura", che discende a sua volta dal "sentimento". L'ambiguità di un termine abusato qual è il sentimento richiede un commento chiarificatore. Nel caso specifico esso rinvia alle nozioni di indole e costume, ricomprese nel più largo concetto di tradizione, ove le sensazioni si concretizzano in prassi creative che attecchiscono senza subire altre pressioni che non siano quelle sociali. Divenute consuetudine esse si trasformano in natura, anzi in seconda natura per adoperare una locuzione cara al Novecento, vera quanto quella ermetica nascosta tra i calcoli. Da questa crasi si forma un paesaggio sonoro commisto di natura autentica e di natura di secondo grado, nel quale si cala l'esperienza individuale di ogni "nazione".

Posto in termini concreti, ma ingenui sotto il profilo filosofico, il quesito di Tartini insidia il dibattito su senso e ragione, diventato stantio in quanto risolto sempre all'interno di un decorso storico che prende le mosse dalla musica greca. La fictio della periodizzazione

19 "Lettera I del sig. co. Giordano Riccati." Sebbene la lettera a Giovenale Sacchi risalga al 31 luglio 1788, quindi 18 anni dopo la scomparsa di Tartini, essa riflette le idee precedentemente esposte al violinista; cfr. l'introduzione di Luca Del Fra al Commercio di lettere [...] Giordano Riccati, XXIV.

${ }^{20}$ I Principes généraux d'acoustique apparvero in Diderot, Mémoires, 23-281. Sull'argomento vedi Gozza e Serravezza, Estetica e musica, 17-19.

${ }^{21}$ Morpurgo Tagliabue, Il gusto nell'estetica, 85-88: nota 425. 
impostata sulla storia di una parte dell'Europa contrastava infatti con le musiche di altri continenti e delle stesse subculture in seno al nostro. Al cospetto di un panorama identitario nuovo, fondato su una visione dinamica del progresso dei popoli, bisognava riscrivere l'enciclopedia dei saperi musicali. Indicativo l'abbozzo della General History of Music di Charles Burney, ricopiato da Giambattista Martini e pensato dall'erudito inglese per accogliere accanto alle civiltà antiche le moderne "nazioni" d'Europa sorte nel medioevo. Nonostante la risoluzione finale, nel settimo capitolo Burney avrebbe voluto discettare "Della musica nazionale italiana, tedesca, francese, inglese, scozzese, irlandese, gallese, russiana, portoghese, spagnuola, africana, asiatica ed americana", come recitano gli appunti di Martini risalenti al 1770, raccolti sotto il titolo di Piano generale per una Storia della musica del dottor Carlo Burney, col catalogo della sua biblioteca musicale. ${ }^{22}$

Sul divario tra categorie in antitesi, la musica studiata secondo le prospettive di ragione e senso o le controversie su natura e cultura verificate grazie al conflitto tra musica colta e musiche altre (extraeuropee, del popolo ingenuo, degli antichi), mi sia concesso citare due atteggiamenti pertinenti alla linguistica e alla musicografia negli anni in cui operò Tartini.

Per quanto il rimando agli studi sulle lingue possa apparire azzardato rispetto al tema in esame, è evidente la similarità di posizioni negli scritti di teoria e storia della musica, avvalorate da un impulso scientista atto a spiegare il mondo mediante assiomi indiscutibili. La vecchia scuola di Port-Royal, per esempio, aveva sancito una separazione fittizia tra ragione e uso, introducendo il discrimine dell'eccezione che non avversa ma conferma la regola. Il primo concetto concerne le norme che presiedono aprioristicamente alla "costituzione grammaticale delle lingue", il secondo si riferisce "a tutto ciò che nel linguaggio umano è accidentale [...] e deviante" rispetto a quella stessa nomotetica. ${ }^{23}$ Nicolas Beauzée, nell'articolo Grammaire dell'Encyclopédie, prende tutt'altro partito. ${ }^{24}$ Egli concede che la "Grammaire générale est [...] la science raisonnée des principes immuables [...] dans toutes les langues", ma la "Grammaire particulière est l'art d'appliquer aux principes immuables [...] les institutions arbitraires et usuelles d'une langue particulière". Ne deriva che "l'usage n'est donc pas le tyran des langues, il en est le législateur naturel, nécessaire et exclusif; [...] et je dirois d'après cela, qu'une langue est la totalité des usages propres à une nation pour exprimer les pensées par la voix". ${ }^{25}$

Alla polemica tra le due scuole di pensiero si possono accostare le idee contrapposte di Tartini e padre Martini in merito al valore di utenza. Il francescano, mitigando in minima misura l'assetto dispersivo della Storia della musica, conferisce all'opera una coesione narrativa mediante il canone dell'armonia che percorre le epoche, nonostante la palese varietà delle forme e degli stili. ${ }^{26} \mathrm{Si}$ tratta di un concetto fragile; un fantasma metastorico coagulante in un unico plesso le proporzioni, il contrappunto medievale e il basso continuo. L'eccessiva importanza conferita all'armonia nasce dallo studio comparativo

${ }^{22}$ Bologna, Museo della Musica, Ms. F 62; facsimile a cura di Vincent Duckles.

${ }^{23}$ Rosiello, "Il pensiero linguistico dell'illuminismo," 203-205.

${ }^{24}$ Encyclopédie ou Dictionnaire raisonné des sciences, des arts et des métiers [1757], t. VII, 842, cito da Rosiello.

${ }^{25}$ Rosiello, "Il pensiero linguistico dell'illuminismo," 203-205.

${ }^{26}$ A titolo di esempio cfr. la terza Dissertazione: "Pregi della musica de' greci e maravigliosi effetti da essa prodotti," in Martini, Storia della musica, 2, 281. 
condotto sulle consonanze e le dissonanze, rimaste pressappoco inalterate nel corso dei tempi. Per cui intorno alla musica d'arte si condensa una visione restrittiva assonante con la Grammaire générale di Port-Royal, se si eccettua il fatto che non si accordano in questo frangente la postura sincronica dei linguisti e quella diacronica del bolognese. Il quale ribadirà lo stesso principio nel 1763 con la premessa alla Synopsis musicarum graecarum atque obscuriorum vocum, aggiunta alla edizione moderna dei trattati di Giambattista Doni (1763): "L'armonia, la natura e proprietà delle consonanze e dissonanze e loro disposizione, come principi ed elementi sono sempre stati e sempre saranno gli stessi, perché fondati sopra le leggi della natura. L'uso però e la loro disposizione [...] varia e si muta secondo la diversità dei tempi.'27 Vent'anni dopo, per restare nel medesimo ambito, John Hawkins predicherà che la sintassi compositiva predispone empiricamente le nozioni dell'armonia, "as the rules of grammar are [drawn] from the speech". ${ }^{28}$ Tartini, invece, mantiene il discorso su basi sincroniche e, dall'uso particolare della grammatica, passa alla verifica della discontinuità culturale che mette in crisi l'idea di un'armonia funzionante come un manipolo di leggi indipendenti dalla volontà degli uomini.

Dal menzionato Conti, il quale sostiene che la quantità delle intonazioni dipende dal clima e dall'indole razziale, a Giambattista Vico, per il quale ogni popolo ha una distinta fisionomia giacché forgia da sé la propria cultura, il tragitto che conduce al pensiero di Tartini è irto di difficoltà. Probabilmente egli ebbe familiarità con le idee del conterraneo, forse incrociò anche la Scienza nuova cara agli intellettuali veneti, e inoltre poteva leggere l'Histoire de la musique di Bonnet-Bourdelot (1715), ove si parla della lontana Cina e della Persia. Nei suoi saggi, purtroppo, non v'è alcun rimando a questa bibliografia, né una rimodulazione tangibile della storiografia passata e coeva.

Dalla tentata fondazione di una verità d'armonia bastevole a sé, il passaggio all'implementazione dello sguardo antropologico corrompe, sino a renderlo inerte, l'iniziale esprit de géométrie propugnato dal violinista. Lo accerta la ripresa del discorso sulla disseminazione del genere diatonico, ove l'analisi empirica non disdegna la meditazione storica, ancorché troppo esile. Nel trattato De' principj dell'armonia musicale contenuta nel diatonico genere (1767) egli si attiene al dato esperienziale, per verificare una straordinaria coincidenza tra verità di "istituzione" e verità di "natura". ${ }^{29} \mathrm{Il}$ genere diatonico, in vigore sin dai tempi dei greci, è presente in qualsiasi "nazione" e assume l'aspetto di un continuum che invalida il ricorso a prove surrettizie tratte dalla speculazione matematica. In esso l'artificio si coniuga felicemente con la natura e vanifica qualsiasi pregiudizio circa un incontro ritenuto impraticabile. Ciò comporta una risignificazione del concetto di natura, da tradursi nel gradimento dovuto alla percezione uditiva, che investe tutti i popoli e resiste allo scorrere del tempo. "La musica attuale", insiste Tartini, "avendo dagli antichi greci la sua primiera istituzione fondata sul diatonico genere, e questo avendo retto per migliaia d'anni alla prova del sentimento di tante diverse nazioni, e di tanti

${ }^{27}$ La sinossi fu richiesta a Martini da Giambattista Passeri per l'edizione dei Trattati di Giovan Battista Doni, dopo l'uscita del primo volume della Storia e avanti la pubblicazione dei due successivi nel 1770 e nel 1781. Cfr. Doni, De’ trattati di musica, 226.

${ }^{28}$ Hawkins, General History of the Science, XLIII.

${ }^{29}$ Tartini, De' principj dell'armonia, 18-19. 
esquisiti professori dell'arte, con ciò porta seco quel tal argomento di verità d'istituzione e di natura". ${ }^{30}$

Il violinista ridefinisce dunque il rapporto di dipendenza dell'arte dalla natura e afferma la naturalità dell'intervento umano, per cui i greci hanno creato il genere diatonico e questo continua ad attecchire ovunque nonostante il mutare delle civiltà. Lo strumento prescelto tra i tanti, il detto diatonico, è anche un dato culturale che risponde alle caratteristiche della musica d'arte e Tartini lo assolutizza in virtù della sua fortuna millenaria. Sulla scia di Ossian, l'argomento rispunta in una lettera di Benjamin Franklin diretta nel 1765 all'amico Lord Kames, ove il concetto di natura coinvolge la musica scozzese rimasta incorrotta e basata sul diatonismo. Analogo, e fatalmente specioso, il ragionamento esposto qualche tempo dopo da John Hawkins nella General History of the Science and Practice of Music (1776): "the ancient Scotch tunes seem to consist of the pure diatonic intervals $[\ldots]$ and in favour of this notion it may be observed that the front row of a harpsichord will give a melody nearly resembling that of the Scots tunes". ${ }^{31}$

Se da un lato il salto di qualità nei Principj è notevole, dall'altro il trattato conferma i limiti di Tartini, ossia la sua inadeguatezza di fronte alle questioni estetiche e l'incapacità di pensare alla musica senza il suggello di un fondamento che abbatta le barriere della storia. Nonostante le rettifiche, rivelatrici di un dubbio critico apprezzabile, nei suoi interventi alligna sempre il credo in un principio dal quale devono discendere le applicazioni più varie. Tuttavia, per dare un esempio di quanto il teorico fosse meno propenso all'eurocentrismo degli intellettuali che iniziavano a occuparsi di altre civiltà, soccorre la questione del canto dei dalmati, da lui sollevata ma sostanzialmente irrisolta. Le implicazioni di metodo contenute nelle relazioni di viaggio di Alberto Fortis e nella risposta di Ivan/Giovanni Lovrić, tutte degli anni Settanta, sono meno originali di quelle fornite da Tartini. Senza scendere in particolari, basterà soffermarsi sul sapido commento di Lovrić in merito all'epica intonata dai morlacchi, ossia gli abitanti della Dalmazia interna, ritenuti autentici slavi del sud in quanto non corrotti dalla cultura italiana:

Per la compiacenza di decantar le gloriose gesta degli antichi eroi della nazione [= illirica o degli slavi del sud, NdA], i morlacchi quasi tutti sono musici. I loro canti eroici sono composti di versi decasillabi, ora rimati a due a due ed ora no. Ciascuno di questi versi è preceduto, cantando, da un lunghissimo oh! con cui pure si chiudono. Le modulazioni della voce ne' canti eroici è rapida. Gli oh!, che sembrano tanti urli de' lupi, sono i trilli. Quando un cantore è accompagnato da qualche strumento i trilli sono flebili.

La gusla è lo strumento principale di musica che si adopera cantando, ed è fatta da una corda sola, composta di molti crini di cavallo uniti insieme. L'arco della gusla ha una corda simile. Pochi sono i morlacchi che sappian suonar a perfezione questo strumento musicale, con cui si pretende di mover qualunque affetto a guisa degli antichi greci; ma chi è assuefatto alla musica italiana non può risentir altro vantaggio che quello di restar attuffato in una rapidissima melanconia.

Se v'è qualche distinto suonatore di gusle è ascoltato con sommo silenzio ed attenzione da' suoi nazionali. Donde proviene ciò? Le loro orecchie armoniche sono talmente

30 Ibid.

${ }^{31}$ Hawkins, General History of the Science, 4. Su questo aspetto cfr. Gelbart, The Invention of “Folk Music”, 115-120. 
organizzate, che per provar il piacer della musica hanno d'uopo di que' suoni, cui li adattò la consuetudine che si cangiò in natura. E che sia vero ciò, la musica italiana li annoia a maggior segno, in quella foggia istessa che la musica morlacca annoia un italiano. [...] Alcune delle loro composizioni hanno un non so che dell'orientale. ${ }^{32}$

Il confronto tra la musica italiana e quella dei morlacchi, proposto dal dalmata Lovrić addentro alle due realtà, statuisce l'intangibilità di una natura intrinseca alla musica occidentale. Deformata dai morlacchi sino al punto da diventare "consuetudine", questa diviene natura altra e acquisisce "un non so che di orientale". Nemmeno il connazionale, quindi, è in grado di giudicare con coerenza i caratteri della tradizione endogena, avendo egli a cuore l'archetipo della musica eurocolta che funge da parametro per qualsiasi espressione.

Con l'Aria del Tasso Tartini abbandona il rigore della teoria per dare un saggio di trasposizione o rilettura del motivo popolare. Della sua passione per la celebre aria si sono occupati parecchi studiosi, ma non è inutile ricomporre i dati raccolti, poiché aiutano a illustrare da un altro punto di vista il complesso intreccio tra popolo e natura. Anzitutto, la sua dedizione al canto popolare, in quanto fenomeno di natura, è attestata dalla curiosità nei confronti degli orbi che a Venezia suonavano il violino nelle calli e si traduce nello studio delle caratteristiche meliche pertinenti all'area demotica ("io in Venezia pagavo il mio trairo a que' tali ciechi suonatori di violino, perché anche da quelli ho imparato molto", lettera a Riccati del 6 febbraio 1760). ${ }^{33}$ Fatto relativamente nuovo per un virtuoso dello strumento che si cimentava con la teoria e non si occupava stabilmente di storia, né tantomeno di estetica. Il suo atteggiamento, comunque lo si voglia classificare, è rivelatore di una mentalità che compulsava anche in tal modo le origini della musica. E il popolare, quale modello di arretramento alla primogenitura, era riguardato come un punto di arrivo accanto agli studi eruditi sulla musica naturalis dell'antichità classica. A scanso di equivoci, io ritengo che le quattro versioni dell'Aria del Tasso offerte da Tartini non facciano parte di un progetto etnografico per fermare sulla carta un canto di popolo altrimenti irripetibile o a rischio di sparizione. La redazione dell'Aria configura piuttosto un'ipotesi di incontro tra una nuova poetica del comporre e l'intuizione antropologica, che vale a conferire dignità alla musica d'arte mediante un riferimento naturale, da non confondere con l'atto del trascrivere allo scopo di tramandare ai posteri.

A seguire il dettato del violinista, il canto di tradizione è rimasto incorrotto non avendo subito l'intervento dei musicisti di professione. Da ciò si dovrebbe dedurre un'assenza poco credibile di permeabilità tra i livelli alto e basso e, a parte questo dettaglio nient'affatto trascurabile, è significativo che egli lasci trasparire nella semplicità dei movimenti lenti di talune sonate un'ispirazione proveniente dal volgo. L'indagine svolta sul campo lo porta a concludere che in qualsiasi civiltà il popolo predilige le cantilene monotone, prive di ornamenti, ossia scevre di quel virtuosismo che solo una mente votata all'arte può realizzare. Ligio al comando che si è imposto nella sua ricerca di verità, preservata dal popolo il quale vive nello stato di natura, egli riproduce più volte l'Aria del Tasso, due versioni della quale presentano una linea melodica assai disadorna. ${ }^{34}$

${ }^{32}$ Lovrich, Osservazioni, 126-127, 131.

${ }^{33}$ Con il numero 9 appare nel citato Commercio di lettere [... Giordano Riccati, 31-32.

${ }^{34}$ Si tratta delle sonate D2, G2, G3, A11; per esempio il terzo movimento della D2 in Re maggiore. 
La pagina del Trattato in cui è esposta l'idea di un canto di popolo privo di modulazioni e povero di ornamenti è stata oggetto di analisi negli studi sulla poetica del compositore. ${ }^{35}$ Tuttavia, non è inutile insistere sulla conversione antropologica di Tartini che si rivela meno scontata di quanto si possa supporre, a partire dalla distinzione operata tra i termini nazione e popolo, quali entità sociali in contatto ma culturalmente divise. Ogni "nazione", scrive senza tentennamenti, "ha le sue canzoni popolari, molte delle quali sono di antica tradizione, molte prodotte di nuovo e adottate dal genio comune". ${ }^{36}$ In merito alla forma delle "canzoni", giudicata una prova della purezza dell'animo incontaminato del volgo che le ha pensate, Tartini sottolinea "che le più semplici e naturali sono le più ricevute". ${ }^{37}$ Quindi può asserire che "il popolo ascolti più volentieri una di queste canzoni di qualunque esquisita cantilena modulata per tutto il suo giro", ed è questa "una osservazione quanto facile a farsi, altrettanto sicura nel verificarsi". ${ }^{38}$ A confutazione si potrebbe immaginare che ciò dipenda da una logica affezione per il testo, e meno per la musica. Ma anche quando un cantante raffinato si prodighi ad abbellire e a complicare la melodia, annota l'istriano, gli ascoltatori incolti prediligerebbero sempre la versione semplice, poiché "la natura ha più forza dell'arte" e "il miglior genere è il diatonico, difficilissimo a ben trattarsi, perché appunto di estrema semplicità, come il più prossimo alla natura". ${ }^{39}$

Anche al di fuori delle teorie è vivo in Tartini il desiderio di fondare parte della propria opera su patterns mutuati dalla natura, ossia dal popolo minuto. Perciò la sua prassi compositiva, ove sia correlata a un vago intendimento etnografico, desta un certo stupore. In specie la serie dei movimenti lenti che, come si è accennato, esibiscono l'intenzione di riprodurre lo spirito lirico della fonte se non proprio la sua texture. Uno dei primi a dare rilievo al processo di scrittura in relazione all'Aria del Tasso fu Charles Burney. Riprendendo il pensiero del violinista, lo storico britannico ripete che l'antica musica degli italiani è a una sola voce ed ha le caratteristiche del recitativo, impossibile a eseguirsi a misura: "According to Tartini, 'the old Italian songs being only made for a single voice, were simple in the highest degree; partaking of the nature of recitative, but largo' as the gondoliers at Venice still sing the stanzas of Tasso". ${ }^{40}$

La citazione non consente di stabilire se Burney intendesse riferirsi a qualche "Italian song" particolare, ma non sarebbe fuori luogo ipotizzare che egli avesse confidenza con le Ottave alla fiorentina e il Tasso alla veneziana pubblicati a Londra da Joseph Baretti nel 1768. Le due formule declamatorie sottomesse ai versi della Gerusalemme, "Intanto Erminia fra l'ombrose piante", furono registrate dal violinista Felice Giardini su richiesta di Baretti, che le inserì nel secondo volume di An Account of the Manners and Customs of Italy con il seguente commento in merito ai gondolieri: "Such stanza's and poems they are

Cfr. Brainard, Le sonate per violino.

${ }^{35}$ Ultimo dei quali Polzonetti, Tartini e la musica secondo natura.

${ }^{36}$ Tartini, Trattato di musica, 243-244.

${ }^{37}$ Ibid.

${ }^{38}$ Ibid.

${ }^{39}$ Ibid.

${ }^{40}$ L'History di Burney fu pubblicata come segue: I 1776, II 1782, III 1789. Cito dal facsimile Dover della seconda edizione in due volumi a cura di Frank Mercer: Burney, A General History of Music, 129. 
very fond of singing, particularly by moon-shine. And as the tune to which they sing them is ancient, and very fine in the opinion of our musicians, my reader cannot be displeased to have it here, as signor Giardini has done me to write it for me". ${ }^{41}$

In un altro capitolo della History Burney prende spunto dallo stile "rustic" delle barcarole, conforme a quello di un canto fermo leggermente elaborato, per immaginare in via retrospettiva che le "terze rime" dantesche fossero cantate con la stessa semplicità: "We may easily image that the music of such singers was not more refined than their pronunciation, and that the melody to which they sung the terze rime of Dante as equally simple and rustic with that to which the gondolieri of Venice still sing the ottave rime or stanzas of Tasso, and which is little more than a species of canto fermo". ${ }^{42}$ Alla "Psalmodie", peraltro, aveva pensato anche Jean-Jacques Rousseau, sulla cui traccia si è mosso Johann Wolfgang Goethe nel 1786, ammaliato dalla esecuzione responsiva in forma di eco tra due cantori in laguna ("Die Melodie, welche wir durch Rousseau kennen, ist eine Mittelart zwischen Choral und Rezitativ"). ${ }^{43}$

A conferma del fascino esercitato dal celebre motivo su Tartini, concorre il fatto che in due casi egli trascrive il testo poetico della Gerusalemme (XII, 36, Lieto ti prendo), ma non si preoccupa di fornire qualche delucidazione sul significato di canto popolare relativamente all'Aria del Tasso. ${ }^{44} \mathrm{~A}$ mio avviso non poteva essere altrimenti. Considerato che tale musica era solo uno dei mezzi probatori per acclarare il significato di natura, essa non poteva ambire all'autonomia semantica del canto di popolo che sarà invocata da poeti e intellettuali alla fine del secolo e perlopiù fuori d'Italia. Per non travalicare dunque il confine segnato dalle testimonianze che trattengono dal pensare a una compiuta formazione del canone popolare, si può riandare alla ben nota voce Barcarolles stilata da Rousseau per il Dictionnaire de musique (1768). ${ }^{45}$ Le barcarole, a dire del filosofo, erano talmente deliziose che nessun compositore poteva sottrarsi al desiderio di imitarne i modi e la condotta melodica. Dubita invece che questo genere sia circonfuso di un'aura demotica, in quanto i gondolieri erano costretti per ragioni di lavoro a frequentare i teatri d'opera e ciò potrebbe avere affinato il loro gusto. Per cui si vede obbligato a precisare che "les airs des barcarolles" sono fatte "pour le peuple" e "souvent composés par les gondoliers mêmes" (corsivi miei). ${ }^{46}$ In entrambi i casi, compositori e gondolieri si attenevano alla semplicità onde imitare la naturalezza di quei canti, e i termini simple e naturel evocati da

${ }^{41}$ Baretti, An Account of the Manners, 153-154.

${ }^{42}$ Burney, A General History of Music, 626-627.

${ }^{43}$ Goethe, Italienische Reise, 84-86, cfr. le ottime considerazioni di Polzonetti, Tartini e la musica secondo natura, 108-109, 118. Non si esclude che a spingere Rousseau a redigere l'articolo sulla barcarola per il Dictionnaire de musique, 60-61, sia stata la lettura e/o la musica di Tartini. Nel manoscritto R68 del filosofo, conservato nella Biblioteca Universitaria di Neuchâtel, compaiono delle annotazioni sul Trattato di musica del 1754, collegate a un appunto inequivocabile: "Barcarole/mot à ajouter," cfr. Eigeldinger, "Rousseau, Goethe," 215.

${ }^{44}$ Polzonetti, Tartini e la musica secondo natura, 102-103, ove sono riportati i quattro incipit.

${ }^{45}$ Rousseau, Dictionnaire de musique, 60-61.

${ }^{46}$ Ibid. Una prova indiretta di trasmissione dal colto al popolare, in merito alle consimili Arie da battello, viene da Marcello, Teatro alla moda, 51. I copisti, scrive il patrizio veneziano, "sapranno comporre, cantare, suonare recitare etc., riducendo la maggior parte dell'arie dell'opera in canzon da battello". 
Rousseau attestano una stretta contiguità con i corrispondenti italiani impiegati da Tartini. Infine il ginevrino avanza il paragone classicistico, che rafforza la mia convinzione circa la parentela istituita nella seconda metà del Settecento tra il canto popolare e i poemi di Omero ancora una volta in nome della nobile semplicità. Rousseau si appella infatti alla fortuna che arrise all'Iliade e all'Odissea grazie agli omeridi, paragonabile solo alla lunga durata del poema di Tasso, intonato par coeur per oltre due secoli. Tuttavia, l'opportunità di approfondire il problema della trasmissione orale, tema cruciale alla fine del secolo, coglie impreparato il filosofo alla pari di Tartini. Se da un lato il ginevrino si avvede della difficoltà a riprodurre in notazione corrente la musique exotique riferita alla Planche $\mathrm{N}$ del Dictionnaire, dall'altro non spende una parola sull'incerta configurazione melodica dei canti dei gondolieri, che ascoltò a Venezia con grande ammirazione nel corso di un intero anno (settembre 1743-agosto 1744). Peraltro è sconfortante l'assenza del popolo alla voce Naturel del citato Dictionnaire. ${ }^{47}$ Ciò preclude l'evenienza di istituire un raffronto con le meditazioni ben più profonde dell'inquieto Tartini, in quanto l'attributo "naturel" è riferibile a qualsivoglia canto "aisé, gracieux, facile", e a qualsiasi armonia quando essa "a peu de renversements" e "dissonances": nulla che ricordi il ricupero del genere diatonico, la cui semplicità è determinante per la sopravvivenza della musica popolare.

Nonostante il divario cronologico e intellettuale non siano lievi, la riflessione di Tartini sulla natura può essere chiosata con le parole di Friedrich Schiller, come aveva felicemente intuito Petrobelli. ${ }^{48}$ La perdita della natura, nel denso Über naive und sentimentalische Dichtung del 1796, provoca una perenne tensione al ritorno. Dopo l'ingresso nello stato di cultura con la formazione della civiltà moderna, il compositore come il poeta sentimentale ambisce a riconquistare lo stato di natura. Il suo tentativo di ristabilire l'armonia infranta dopo il declino della civiltà classica avviene per il tramite del popolo, il cui canto è l'espressione concreta dell'ingenuità. Schiller rintraccia l'ingenuo "in den Sitten des Landvolks und der Urwelt" ("nei costumi del popolo contadino e nel mondo primitivo"), in quanto "Sie sind, was wir waren; sie sind, was wir wieder werden sollen" ("essi sono ciò che noi eravamo; sono ciò che noi dovremo tornare ad essere").49 Anche se il filosofo tedesco non chiama in causa la musica, pure nel suo caso cultura e arte hanno un valore simile a quello attribuito al termine artificio da Tartini. ${ }^{50} \mathrm{Il}$ quale, consapevole dell'assenza di natura nella tradizione artistica da lui stesso praticata, è costretto a riguadagnarla fuori dai luoghi abituali in cui ha operato. Per dirla con le parole di Schiller: "Der Dichter, sagte ich, ist entweder Natur, oder er wird sie suchen. Jenes macht den naiven, dieses den sentimentalischen Dichter", il poeta "o è natura o la cercherà". ${ }^{51} \mathrm{E}$ Tartini ha trascorso buona parte della sua laboriosa esistenza a cercare la natura. Dapprima ha creduto di trovarla nei principi che reggono

${ }^{47}$ Rousseau, Dictionnaire, 503-504.

${ }^{48}$ Petrobelli, Tartini, le sue idee e il suo tempo, 21.

${ }^{49}$ Schiller, Über naive und sentimentalische Dichtung.

${ }^{50}$ Ibid.: "Ist der Mensch in den Stand der Kultur getreten, und hat die Kunst ihre Hand an ihn gelegt, so ist jene sinnliche Harmonie in ihm aufgehoben, und er kann nur noch als moralische Einheit, d. h. als nach Einheit strebend sich äußern. Die Uebereinstimmung zwischen seinem Empfinden und Denken, die in dem ersten Zustande wirklich statt fand, existiert jetzt bloß idealisch; sie ist nicht mehr in ihm, sondern außer ihm, als ein Gedanke, der erst realisiert werden soll, nicht mehr als Thatsache seines Lebens".

${ }^{51}$ Ibid. 
l'universo sonoro, poi nella coesistenza delle culture, ossia nelle musiche delle "nazioni" e del popolo, le quali, con il contributo dei lacerti greci, hanno scalfito la sua fede nella sola ragione. Da quel momento il termine natura è diventato polivalente, persino ambiguo, ma è grazie a questa incoerenza che l'istriano può collocarsi tra i fautori di una poetica antropologica di cui erano ignari i suoi coetanei.

\section{Bibliografia}

Badaloni, Nicola. Antonio Conti. Un abate libero pensatore tra Newton e Voltaire. Milano: Feltrinelli, 1968.

Baretti, Joseph. An Account of the Manners and Customs of Italy with Observations on the Mistakes of Some Travellers with Regard to that Country, 2. London: Davies, 1768. Brainard, Paul. Le sonate per violino di G. Tartini: catalogo tematico. Padova: Accademia Tartiniana; Milano: Carisch, 1975.

Burney, Charles. A General History of Music from Earliest Ages to the Present Period, 2. London: Payne, 1789.

Cavallini, Ivano. "Genio, imitazione, sentimentale e patetico. Gianrinaldo Carli e Tartini: le prospettive della critica tartiniana nella seconda metà del Settecento.” In Tartini. Il tempo, le opere, a cura di Andrea Bombi e Maria Nevilla Massaro, 229-246. Bologna: Mulino, 1994.

Commercio di lettere intorno ai principj dell'armonia fra il signor Giuseppe Tartini ed il co. Giordano Riccati, a cura di Luca Del Fra. Lucca: LIM, 2007.

Conti, Antonio. Prose e poesie, 2. Venezia: Pasquali, 1756.

Descartes, René. Discorso sul metodo. Firenze: La Nuova Italia, 1932.

Diderot, Denis. Mémoires sur différents sujets de mathématiques. Paris: Durand et Pissot, 1748 [Edizione moderna in Euvres completes, 2, a cura di Jean Mayer, 235-281. Paris: Hermann, 1975.]

Doni, Giovan Battista. De’ trattati di musica [...] aggiuntovi un lessico delle voci musiche e l'indice generale, per opera e studio del p. maestro Gio. Battista Martini. Firenze: Stamperia Imperiale, 1763.

Eigeldinger, Jean-Jacques. "Rousseau, Goethe et les barcarolles vénitiennes: tradition musical et topos littéraire.” Annales de la Société J.-J. Rousseau 40 (1992): 213-263. Gelbart, Matthew. The Invention of "Folk Music" and "Art Music". Emerging Categories from Ossian to Wagner. New York: Cambridge University Press, 2007.

Gronda, Giovanna. “Conti, Antonio (Schinella).” In Dizionario Biografico degli Italiani, 28, 352-359. Roma: Istituto della Enciclopedia Italiana, 1983.

Goethe, Johann Wolfgang. Italienische Reise. In Hambuger Ausgabe von Goethes Werken, 11, a cura di Herbert von Einem, 10. München: DTV, 1995.

Gozza, Paolo e Serravezza, Antonio. Estetica e musica. L'origine di un incontro. Bologna: CLUEB, 2004.

Hall, Albert Rupert. "La matematica, Newton e la letteratura.” In Scienza e letteratura nella cultura italiana del Settecento, a cura di Renzo Cremante e Walter Tega, 2-46. Bologna: Mulino, 1984. 
Hawkins, John. General History of the Science and Practice of Music. London: Payne \& Son, 1769-1776.

Leydi, Roberto. "Erminia monta in gondola." In Tartini. Il tempo, le opere, a cura di Andrea Bombi e Maria Nevilla Massaro, 417-442. Bologna: Mulino, 1994.

"Lettera I del sig. co. Giordano Riccati intorno al risorgimento della musica: all'onoratissimo padre d. Giovenale Sacchi professore d'eloquenza nel Collegio Imperiale di Milano.” In Continuazione del Nuovo Giornale de’ Letterati 41 (1789): 170-180.

Lovrich, Giovanni/Ivan. Osservazioni [...] sopra diversi pezzi del Viaggio in Dalmazia del signor abate Alberto Fortis coll'aggiunta della vita di Soçiviza. Venezia: Zamboni, 1776.

Marcello, Benedetto. Il teatro alla moda. Venezia: [s. e.], 1720.

Marošević, Grozdana. “Croatia: Traditional Music.” In Grove Music Online, Oxford Music Online. Accesso il 4 febbraio 2014. (http://www.oxfordmusiconline.com).

Martini, Giambattista. Piano generale per una Storia della musica del dottor Carlo Burney, col catalogo della sua biblioteca musicale. Bologna: Museo della Musica, Ms. F 62. [Facsimile a cura di Vincent Duckles. Bologna: AMIS, 1972].

_—_. Storia della musica, 2. Bologna: Dalla Volpe, 1770.

Morpurgo Tagliabue, Guido. Il gusto nell'estetica del Settecento, a cura di Luigi Russo e Giuseppe Sertoli. Palermo: Centro Internazionale Studi di Estetica, 2002.

Newton, Isaac. Optice: sive de reflexionibus, refractionibus, inflexionibus et coloribus lucis libri tres. Londini: Smith et Walford, 1706.

Petrobelli, Pierluigi. "Tartini and Folk Music." In Report of the Tenth Congress of the International Musicological Society. Ljubljana 1967, a cura di Dragotin Cvetko, 176-181. Kassel, Basel, Paris, Ljubljana: Bärenreiter, 1970.

___ Tartini, le sue idee e il suo tempo. Lucca: LIM, 1992.

Polzonetti, Pierpaolo. Tartini e la musica secondo natura. Lucca: LIM, 2001.

Rosiello, Luigi. "Il pensiero linguistico dell'illuminismo.” In Lezioni sull'illuminismo, 203-221. Milano: Feltrinelli, 1980.

Rousseau, Jean-Jacques. Dictionnaire de musique. Paris: Duchesne, 1768.

Rupert Hall, Albert. "La matematica, Newton e la letteratura." In Scienza e letteratura nella cultura italiana del Settecento, a cura di Renzo Cremante e Walter Tega, 24-46. Bologna: Mulino, 1984.

Schiller, Friedrich. Über naive und sentimentalische Dichtung (1794-1796). (Disponibile online: gutenberg.spiegel.de/buch/3347/1).

Tartini, Giuseppe. Trattato di musica secondo la vera scienza dell'armonia. Padova: Manfrè, 1754. [acura di Enrica Bojan, Palermo: Novecento, 1996].

___ De' principj dell'armonia musicale contenuta nel diatonico genere. Padova: Stamperia del Seminario, 1767. 


\author{
NARAVA IN DRUGAČNOST \\ NEKAJ OPOMB O ARII DEL TASSO GIUSEPPA TARTINIJA
}

\title{
Povzetek
}

Poseben odnos Giuseppa Tartinija do ljudske glasbe je čutiti v njegovem celotnem opusu. Kar štiri stavke svojih violinskih sonat je osnoval na priljubljeni temi Arije del Tasso, ki je vzburila tudi pozornost J. J. Rousseauja in J. W. Goetheja v času njunega bivanja v Benetkah.Tartini se je kot skladatelj in teoretik skušal približati naravi. Z zanimanjem glasbenega narodopisca je zapisoval pesmi beneških gondoljerjev ali pa jih je po svoje prilagajal. V njegovi interpretaciji je beseda ljudsko (it. popolare), skupaj s t. i. musico naturalis starih Grkov in »glasbo narodov«, treba razumeti kot preprosto in preprostost je glavna značilnost narave. V svojih spisih je besedo pogosto uporabljal kot nasprotje koncepta umetnega (it. artificioso), ki se prevaja kot umetelno ali nespontano, in tak koncept narave je v svojem pomenu v obdobju med 1754 in 1767 doživel pomembne spremembe.

Tartini, ki je seveda sledil znanstvenim metodam svojega časa, se naslanja na harmonske osnove, povezane z matematiko; po eni strani na stare numerične osnove glasbene akustike in po drugi na akustične poskuse po zgledu Rameauja (npr. v Trattato di musica secondo la vera scienza dell'armonia, 1754). Tako harmonijo označuje not naravni fenomen, prežet z univerzalnostjo, ki je osnova tako tradicionalne kot tudi »nacionalne« glasbe. Čeprav se ljudska in umetna glasba izražata v različnih oblikah, verjame, da ratio harmonije v obeh pojavnostih vlada enako, tako kot tudi splošna slovnična pravila enako veljajo za različne jezike (v tem primeru ratio sovpada s sensusom). Ne glede na povedano pa se že v nizu pisem, ki jih je naslovil na matematika Giordana Riccatija (Commercio di lettere sui Principj dell'armonia), kaže dvom v prejšnje ideje, kot rezultat nekompatibilnosti med ljudsko in umetno glasbo oziroma med umetno glasbo in tisto, ki jo imenuje »glasba narodov«. Tartini sprejema raznolikost glasbenih oblik, sobivajočih v okviru vzporedne druge narave, in tako drugačnost razume kot pojavno kategorijo. $\mathrm{V}$ tem smislu »harmonija« ne more vsebovati dveh ločenih glasbenih domen ali razlagati njune skupne drugačnosti. Še več, iz te dvojnosti nujno sledi trk ratia in sensusa, ki njuno dvojnost skoraj spreminja v naravo in čustvovanje.

To spoznanje, ki sega v šestdeseta leta 18. stoletja, je istrskega skladatelja napeljalo $\mathrm{k}$ iskanju poti, kako predstaviti naravo s pomočjo diatonike. Ne da bi se odpovedal ideji skupnega faktorja, je v diatoniki starogrške glasbe videl arhetip, ki se pojavlja kot skupni element v ljudski pesmi, starih napevih in umetni glasbi. Kot je zapisal v svojem delu $D e^{\prime}$ principj dell'armonia musicale contenuta nel diatonico genere (1767), je prav diatonika tista osnova, ki se je še najbolj neokrnjeno ohranila skozi čas in prostor.

Čeprav Tartini ni dodelal svoje estetske teorije, ki bi podpirala njegove trditve, je očitno, kako opisani antropološki pogled spodkopava njegove prejšnje racionalistične koncepte narave. $\mathrm{V}$ tem pogledu s svojim vprašanjem že naznanja razprave o »ljudski« glasbi in njenem pomenu, ki so se razvile šele od sedemdesetih let 18. stoletja dalje. 